\title{
RAMON M. ARANA
}

\section{Programming with parametric elements} of the matrix coefficients

\author{
Revue française d'automatique, d'informatique et de recherche \\ opérationnelle. Recherche opérationnelle, tome 11, n 2 (1977), \\ p. 233-238. \\ <http://www.numdam.org/item?id=RO_1977_11_2_233_0>
}

(C) AFCET, 1977, tous droits réservés.

L'accès aux archives de la revue « Revue française d'automatique, d'informatique et de recherche opérationnelle. Recherche opérationnelle » implique l'accord avec les conditions générales d'utilisation (http://www.numdam.org/ legal.php). Toute utilisation commerciale ou impression systématique est constitutive d'une infraction pénale. Toute copie ou impression de ce fichier doit contenir la présente mention de copyright.

\section{Numdam}

Article numérisé dans le cadre du programme

Numérisation de documents anciens mathématiques

http://www.numdam.org/ 


\title{
PROGRAMMING WITH PARAMETRIC ELEMENTS OF THE MATRIX COEFFICIENTS
}

\author{
by Ramon M. Arana
}

\begin{abstract}
Many studies have been carried out concerning parametric programming of the objective function coefficients and also the parametric programming of the constant terms of the constraints has been extensively studied

However, many authors state that parametric programming of elements of the matrix coefficients is simple and useful only when the coefficients that vary correspond to nonbasic vectors, and that when, on the other hand, they correspond to basic vectors the complexity of the topic does not make this type of parametric programming very useful

In this article one obtains a formula which determines the critical values of a parameter which affects the coefficients of a basic vector While the parameter does not reach these limits, the new vector continues forming a feasible basis with the remaining vectors of the optimal basis

Five useful examples of parametric programming of elements of the matrix coefficients have been given in 1963 by Henri Maurin [1] with determination of the critical values of the parameter
\end{abstract}

\section{INTRODUCTION}

Many studies have been carried out concerning parametric programming of the objective function coefficients and also the parametric programming of the constant terms of the constraints has been extensively studied.

However, many authors state that parametric programming of elements of the matrix coefficients is simple and useful only when the coefficients that vary correspond to nonbasic vectors, and that when, on the other hand, they correspond to basic vectors the complexity of the topic does not make this type of parametric programming very useful.

In this article one obtains a formula which determines the critical values of a parameter which affects the coefficients of a basic vector. While the parameter does not reach these limits, the new vector contınues forming a feasible basis with the remaining vectors of the optimal basis.

Five useful examples of parametric programming of elements of the matrix coefficients have been given in 1963 by Henri Maurın [1] with determınation of the critical values of the parameter. 


\section{NOTATIONS}

Let us consider a problem of lineal programming presented in the following manner :

Minimize $z=c x$, subject to:

$$
\begin{array}{r}
A x=b \\
x \geqslant 0
\end{array}
$$

Let us designate by $a_{i j}$ the element of the matrix $A$ located in row $i$ and in column $j$. The matrix $A$ is composed of $m$ rows and $n$ columns.

Let $B$ be the matrix whose columns are the $m$ vectors of the optimal basis. Let us suppose that the $m$ vectors of the optimal basis correspond to the $m$ first columns of the matrix $A\left({ }^{1}\right)$.

Let $B^{-1}$ be the inverse matrix of $B$.

The solution of the problem of linear programming is defined by the following formula :

$$
x_{B}=B^{-1} b \text {. }
$$

The vector $a_{j}$ can be expressed, as a linear combination of the vectors that form the basis $B$, in the following way:

$$
a_{j}=B y_{j} \quad y_{j}=B^{-1} a_{j} .
$$

Let us designate by $\beta_{i}$ the row vector composed of the elements of row $i$ of the matrix $B^{-1}$.

\section{PARAMETRIC PROGRAMMING OF A BASIC VECTOR}

Let us suppose that $a_{h}$ is the vector that will be affected by the parameter $\theta$. Since this vector belongs to the optimal basis (calculated for $\theta=0$ ), it results that :

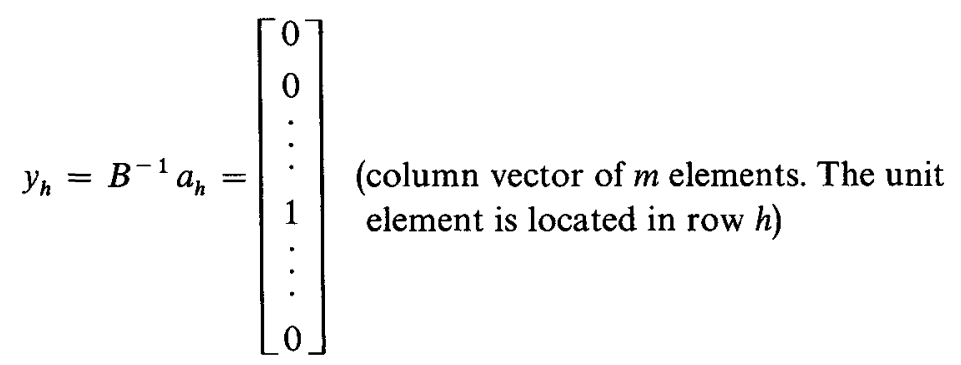

$y_{h}$ is a column vector of $m$ elements, all of which are zero, except one element, located in row $h$, whose value is 1 . 
Let us suppose that vectors $y_{t}$ corresponding to the calculated optimal basis are organized in such a way so that the unit element of the column vector $y_{t}$ would be located in row $i\left({ }^{2}\right)$.

In order to perform the parametric analysis one substitutes $a_{h}$ for $a_{h}^{\prime}$. Let us suppose that between $a_{h}$ and $a_{h}^{\prime}$ there exists the linear function defined by the following expression :

$$
a_{h}^{\prime}=a_{h}+\theta e \quad e=\left[\begin{array}{c}
e_{1} \\
e_{2} \\
\vdots \\
e_{m}
\end{array}\right]
$$

Then :

$$
y_{h}^{\prime}=B^{-1} a_{h}^{\prime}=\left[\begin{array}{c}
\theta \beta_{1} e \\
\theta \beta_{2} e \\
\vdots \\
1+\theta \beta_{h} e \\
\vdots \\
\theta \beta_{m} e
\end{array}\right]
$$

\section{GEOMETRIC INTERPRETATION}

In linear programming problems reasonings must be developed in a space of $m$ dimensions, where $m$ can be larger than 3 .

The graphic representations can only be made in a space of, as a maximum, 3 dimensions.

The principal difficulty in obtaining, by geometric means, deductions applicable to the problems of linear programming, consists in that only those deductions obtained in a space of 3 dimensions which are also valid in an $m$-dimensional space are useful.

However, on passing from the space of 3 dimensions to that of $m$ dimensions, certain concepts that were perfectly clear and understandable, and that were also amenable to an easy mental representation, become abstract ideas, much more complex.

In spite of everything, geometric reasoning is at times an efficient method of investigation, or at least a means which permits one to deduce subjects which are to be the object of investigation.

\footnotetext{
$\left({ }^{1}\right)$ and $\left({ }^{2}\right)$ : These suppositions simplify the notations, and do not dimınish the general character of nether reasonings nor deductions.
} 
The non-negativity restrictions of the variables requires that the solution vector $x_{B}$ be contained in the polyhedral convex cone determined by vectors $y_{i}$ which form the basis.

Therefore, if in a feasible basis one of the vectors varies, the modified basis will continue to be feasible basis as long as vector $x_{B}$ be contained in the polyhedral convex cone determined by the vectors that form the new basis.

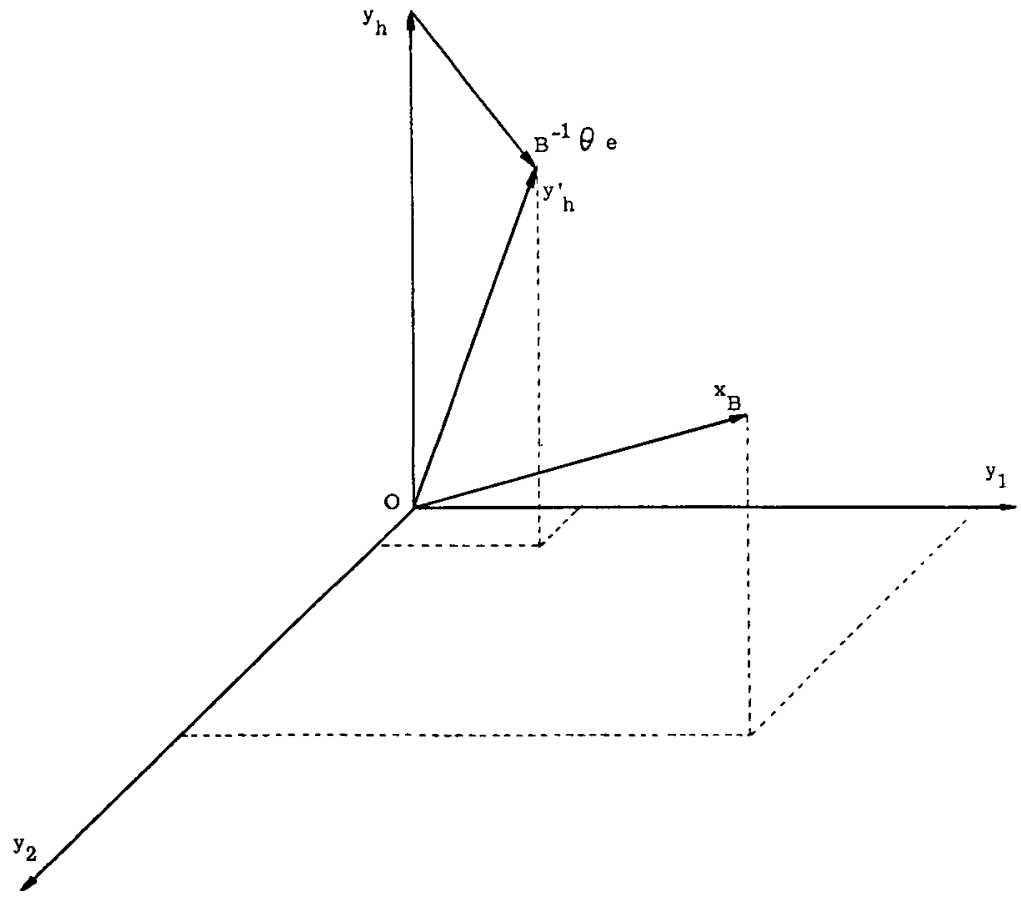

Let us consider a space of 3 dimensions, and let us take as the axis the right lines that contain the vectors $y_{i}=B^{-1} a_{\imath}$ that form the basis.

Let us represent vector $x_{B}=B^{-1} b$, vector $y_{h}^{\prime}=B^{-1} a_{h}^{\prime}$ and the variable vector $B^{-1} \theta e$ (and remember that $y_{h}^{\prime}=B^{-1} a_{h}+B^{-1} \theta e$ ).

$$
y_{h}
$$

In order that the vectors $y_{1}, y_{2}$ and $y_{h}^{\prime}$ form a feasible basis it is necessary that vector $x_{B}$ be contained in the polyhedral convex cone determined by these three vectors.

On considering the plane that contains vectors $y_{h}$ and $y_{1}$ and the plane which contains $y_{h}^{\prime}$ and $y_{1}$, the end of vector $x_{B}$ must be on the same side with respect to both planes. 
Likewise, on considering the plane that contains vectors $y_{h}$ and $y_{2}$ and the plane which contains $y_{h}^{\prime}$ and $y_{2}$, the end of vector $x_{B}$ must be on the same side with respect to both planes.

\section{DEDUCTIONS IN THE $M$-DIMENSIONAL SPACL}

The previous reasoning can be extended to the space of $m$ dimensions $(m>3)$ if, instead of considering the planes which contain vector $y_{h}$ and another vector of the basis (and the planes which contain vector $y_{h}^{\prime}$ and this other vector of the basis), one considers the hyperplanes which contain vector $y_{h}$ and the remaining vectors of the basis except one (and the hyperplanes which contain vector $y_{h}^{\prime}$ and those same remaining vectors of the basis).

The equation of the hyperplane that contains vector $y_{h}^{\prime}$ and all of the vectors of the previous basis except vector $y_{h}$ and another vector $\left(y_{i}\right)$ is :

$$
\begin{gathered}
\frac{y_{i}}{y_{h}}=\frac{\theta \beta_{i} e}{1+\theta \beta_{h} e} \\
y_{i}\left(1+\theta \beta_{h} e\right)-y_{h} \theta \beta_{i} e=0
\end{gathered}
$$

The vector $x_{B}$ (in the case of a non-degenerate solution) has a positive component $x_{i}$.

In order that the modified basis be a feasible basis, the end of $x_{B}$ must be on the side of the positive values of $y_{i}$, with respect to the hyperplane which contains all of the vectors of the previous basis except $y_{i}$, as well as with respect to the hyperplane that contains vector $y_{h}^{\prime}$ and all the vectors of the previous basis except $y_{h}$ and $y_{i}$.

Consequently, it must be satisfied that :

$$
x_{i}\left(1+\theta \beta_{h} e\right)-x_{h} \theta \beta_{i} e \geqslant 0
$$

where $x_{i}$ and $x_{h}$ are the components of vector $x_{B}$ corresponding to rows $i$ and $h$ respectively.

The limit (or limits) of the parameter is obtained by setting the first term of the above inequality to zero, and solving $\theta$.

$$
\theta=\frac{x_{i}}{x_{h} \beta_{i} e-x_{i} \beta_{h} e}
$$

\section{CRITICAL VALUES OF THE PARAMETER}

The inequality (3) must be fulfilled for all values of $i$ corresponding to the vectors that form the calculated optimal basis (calculated for $\theta=0$ ).

In the particular case $i=k$, the inequality (3) is satisfied for any finite value of $\theta$.

Therefore, from the formula (4), one obtains $m-1$ values of $\theta$. 
Some of these values may be positive and others negative.

In the case in which $\theta=0$, the calculated optimal basis is, of course, feasible basis. As we increase the value of the parameter, the modified basis continues being feasible basis until $\theta$ reaches the lowest value of the positive values obtained in the formula (4). Starting from this value, for greater values of the parameter, the previous optimal basis no longer is a feasible basis.

On decreasing the value of the parameter starting from $\theta=0$, another limiting value is reached in the maximum value of those negative obtained in the formula (4).

The critical values of the parameter are, therefore, the lowest of the positive values and the highest of the negative values obtained from the formula (4).

If all of the values of $\theta$ obtained from the formula (4) are of the same sign, the modified optimal basis continues being a feasible basis for any finite value of $\theta$ of the opposite sign.

\section{CASE IN WHICH THE MODIFIED BASIS NO LONGER IS A BASIS}

It can occur also that, on varying one of the vectors of the optimal basis, the transformed previous basis no longer is a basis.

This will occur when the vector affected by the parameter becomes a linear combination of the remaining vectors of the previous optimal basis.

In order for this to occur one must nullify $y_{h}^{\prime}$. That is to say, it will occur that :

$$
1+\theta \beta_{h} e=0 .
$$

Therefore, in the case in which $\theta=-\frac{1}{\beta_{h} e}$, the modified optimal basis ceases being a basis.

\section{FULFILLMENT OF THE OPTIMALITY CONDITION}

The formula (4) determines the limits of $\theta$ between which the modified optimal basis continues being a feasible basis.

Naturally, although one may know that a modified basis is a feasible basis, one must check to see also if it fulfills the optimality condition, by previously substituting in the basis the vector $y_{h}$ for the $y_{h}^{\prime}$. The components of these vectors are determined by the formulas (1) and (2) respectively.

\section{BIBLIOGRAPHY}

1. H. Maurin, Paramétrisation Générale d'un Programme Linéaire (Thèse présentée le 19/6/1963 à la Fac. des Sciences de l'Université de Paris). 